Document downloaded from:

http://hdl.handle.net/10251/100043

This paper must be cited as:

Oroval, M.; Coll Merino, MC.; Bernardos Bau, A.; Marcos Martínez, MD.; Martínez-Máñez, R.; Shchukin, DG.; Sancenón Galarza, F. (2017). Selective Fluorogenic Sensing of As(III) Using Aptamer-Capped Nanomaterials. ACS Applied Materials \& Interfaces. 9(13):1133211336. doi:10.1021/acsami.6b15164

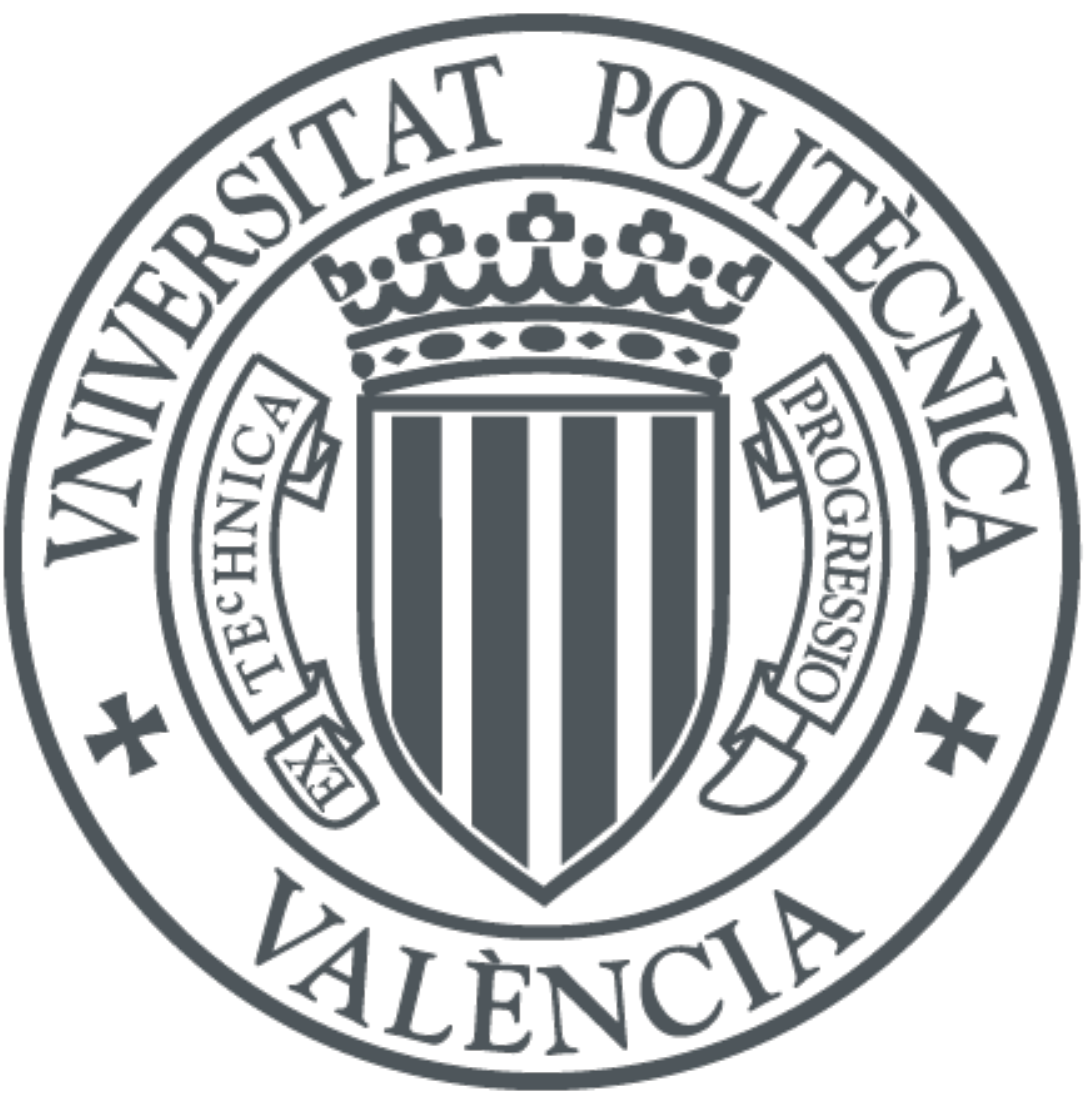

The final publication is available at

https://doi.org/10.1021/acsami.6b15164

Copyright American Chemical Society

Additional Information 


\section{Selective fluorogenic sensing of As(III) using}

\section{aptamer-capped nanomaterials}

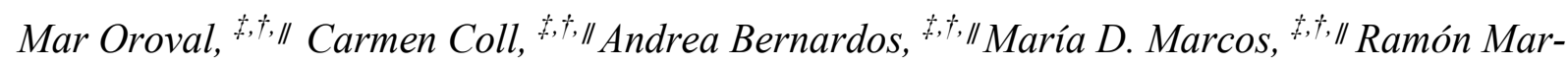

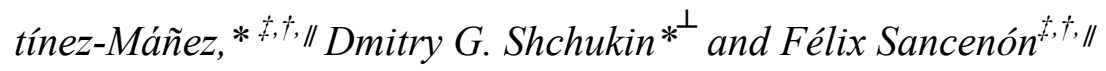

$\$$ Instituto Interuniversitario de Investigación de Reconocimiento Molecular y Desarrollo Tecnológico. Unidad Mixta Universitat Politècnica de València - Universitat de València. Camino de Vera s/n, 46022, Valencia, Spain.

†. CIBER de Bioingeniería, Biomateriales y Nanomedicina (CIBER-BBN).

॥· Departamento de Química, Universitat Politècnica de València, Camino de Vera s/n, 46022, València, Spain.

$\stackrel{\perp}{~}$ Chemistry Department, Stephenson Institute for Renewable Energy, The University of Liverpool, Chadwick Building, Peach Street, Liverpool, L69 7ZF (UK).

KEYWORDS: Hybrid materials, sensing, silica nanoparticles, arsenic, nanomaterials

ABSTRACT. Organic-inorganic hybrid nanomaterials offer extremely valuable tools for monitoring many types of analytes in solution. Within this framework, aptamer-based 
nanomaterials for heavy metal detection are still very scarce. Herein, a novel sensing nanoprobe for the selective and sensitive detection of As(III) based on the combination of aptamers with mesoporous silica nanoparticles has been developed. The efficiency of the sensor is demonstrated in environmental conditions, showing a great potential in As(III) monitoring assays.

Arsenic poisoning is becoming a severe worldwide threat to human health, due to the numerous diseases, including high risk of cancer, associated with drinking water contaminated with arsenic. ${ }^{1}$ The U.S. Environmental Protection Agency (EPA) recently readjusted the maximum contaminant level for arsenic in drinking water from $50 \mu \mathrm{g} / \mathrm{L}$ to $10 \mu \mathrm{g} / \mathrm{L}{ }^{2}$ This change motivated many researchers to develop new sensitive techniques for monitoring arsenic that can accomplish with the new standard. ${ }^{3}$ The predominant forms of arsenic found in aqueous environments are arsenite $\left(\mathrm{As}(\mathrm{III})\right.$ as $\mathrm{H}_{3} \mathrm{AsO}_{3}$, ) and arsenate $\left(\mathrm{As}(\mathrm{V})\right.$ as $\left.\mathrm{H}_{3} \mathrm{AsO}_{4}\right) .{ }^{4}$ Between these two most common forms of arsenic species in water, As(III) has been identified as the most harmful and in fact it is 100 times more toxic than $\mathrm{As}(\mathrm{V}) .{ }^{5}$ Traditional methods for arsenic detection are atomic fluorescence spectrometry, atomic absorption spectrometry and inductively coupled plasma optical emission spectrometry. ${ }^{6,7}$ Although, these methods can accurately measure arsenic, most of them require expensive and sophisticated equipment accompanied with complex sample pre-treatments. In this scenario, the development of simple, sensitive and reliable procedures for As(III) sensing in drinking water becomes to be a necessity. Very recently, as an alternative to these traditional methods, chromogenic and fluorogenic chemosensors for an accurate and sensitive As(III) detection using quantum dots, ${ }^{8}$ cysteine- 
functionalized tetraphenylethenes ${ }^{9}$ and coumarin-appended benzothiazolines ${ }^{10}$ have been described.

In this context, nanostructured sensors have been reported to offer many advantages, to such an extent, that they can be considered as the new generation of sensing systems able to provide rapid and sensitive responses. ${ }^{11}$ Compared with other potential nanomaterials used in sensing protocols, mesoporous silica nanoparticles (MSNs) are ones of the most promising supports due to their remarkable properties such as high inner surface area and flexible surface-modification chemistry. ${ }^{12-14}$ Moreover, MSNs can be functionalized with (bio)chemical or supramolecular ensembles than acted as "molecular gates" for the development of stimuli-responsive systems able to release an entrapped cargo due to the presence of certain and well defined stimuli. ${ }^{15,16}$ These capped materials have been recently applied in sensing protocols. For this purpose, gated MSNs are loaded with a reporter which is selectively delivered to the solution in the presence of a target analyte..$^{13,17}$

On the other hand, the development of sensory systems using aptamers has attracted a great interest to detect trace amounts of many different type of analytes. ${ }^{18}$ Aptamers are DNA sequences with long-term stability, high affinity and selectivity for target proteins, small molecules or ions. ${ }^{18-20}$ Aptamer production is based on a selection method called SELEX (Systematic Evolution of Ligands by Exponential enrichment) and their use in the sensing field has resulted in the design of ultrasensitive systems to detect a number of different analytes. In the field of gated chemistry, aptamers-containing materials for the recognition of ATP, potassium, adenosine and thrombin have been recently reported. ${ }^{21-24}$

Taking into account the above mentioned facts we envisioned the use of aptamer-capped MSNs as potential supports for the design of As(III) sensing systems. Our proposed sensing 
paradigm is showed in Scheme 1. As inorganic scaffold, MCM-41 mesoporous silica nanoparticles $(c a .100 \mathrm{~nm})$ were selected due to their remarkable features for the design of capped materials. ${ }^{25}$ The pores of the MCM-41 inorganic support were loaded with rhodamine B and then the external surface was functionalized with aminopropyl moieties. The final capped solid was prepared by addition of an As(III) aptamer. The presence of As(III) is expected to induce unblocking of the pores, via an effective displacement of the aptamer from the MSNs surface, with subsequent dye delivery.

MCM-41 nanoparticles were obtained in alkaline media using previously described procedure. ${ }^{26}$ To prepare $\mathbf{S 1}$ solid, calcined MCM-41 was suspended in a solution of rhodamine B in acetonitrilie and the mixture was stirred to achieve the maximum loading of the pores. Afterwards, (3-aminopropyl)triethoxysilane (APTES) was grafted onto the external nanoparticles' surface to produce S1 (see Supporting Information for details). To cap the pores we used a 100-mer DNA aptamer (Ars-3) which possesses a very high affinity to As(III) $\left(K_{d}=\right.$ $7.05 \mathrm{nM}) .{ }^{27}$ This aptamer has been used for the detection of As(III) using gold nanoparticles ${ }^{28-31}$ but, as far as we know, the use of Ars-3 in gated-hybrid mesoporous materials has not been reported. Addition of Ars-3 aptamer to a suspension of S1 in HEPES buffer (pH 7.2) resulted in the capping of the pores by means of electrostatic interactions between the positively charged aminopropyl moieties and the negatively charged aptamer, yielding the final material S1-Ars-3 (Scheme 1 and see Supporting Information for detailed description). 


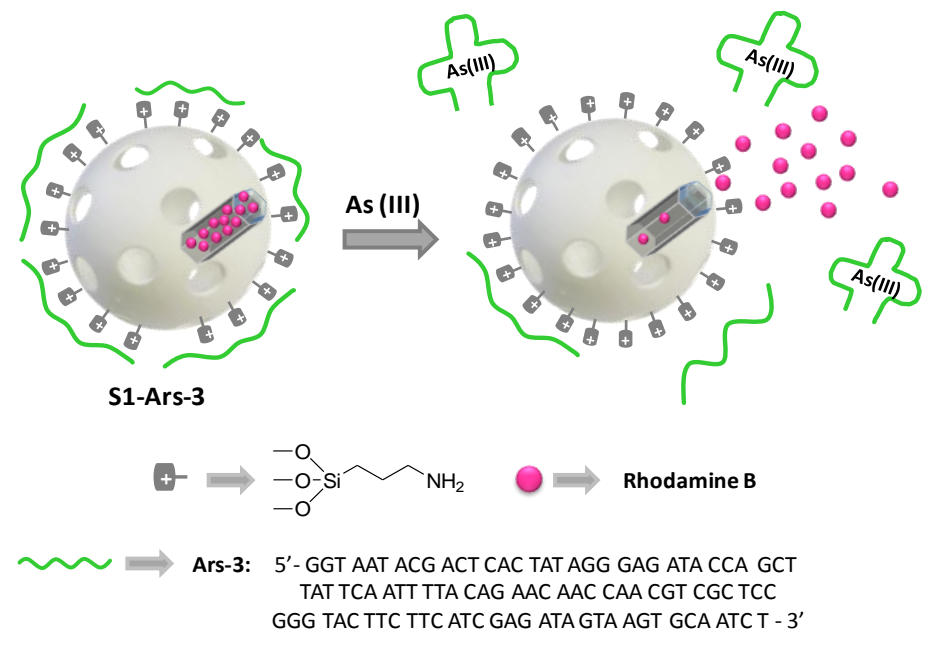

Scheme 1. Representation of the gated material S1-Ars-3 functionalized with aminopropyl moieties and capped with Ars-3 aptamer. Delivery of the entrapped dye (rhodamine B) is specifically accomplished in the presence of As(III).

The starting MCM-41 mesoporous nanoparticles and solid S1 were characterized following standard procedures (see Supporting Information for details). Powder X-ray diffraction (PXRD) and transmission electron microscopy (TEM), carried out on the starting MCM-41 mesoporous nanoparticles, clearly showed the presence of a mesoporous structure that persisted in solid S1 regardless of the loading process with rhodamine $\mathrm{B}$ and further functionalization with (3aminopropyl)triethoxysilane (see Figure 1). The size of the prepared nanoparticles was assessed by TEM images, which gave an average particle diameter of $c a .100 \pm 13 \mathrm{~nm}$ (see also Figure 1). BET specific surface area, pore volume and pore size were calculated from the $\mathrm{N}_{2}$ adsorptiondesorption measurements. Table 1 summarizes the most relevant structural features obtained from these studies. Particle diameter (studied by DLS in HEPES buffer at $\mathrm{pH}$ 7.2) increased after each surface modification. Diameters of 123.5, 165.1 and $190.0 \mathrm{~nm}$ were found for MCM-41, S1 and S1-Ars-3, respectively. Moreover, from elemental analysis and thermogravimetric studies 
the contents of rhodamine B and aminopropyl moieties in solid S1 were calculated to be 0.076 and $2.729 \mathrm{mmol} / \mathrm{g} \mathrm{SiO}_{2}$, respectively.
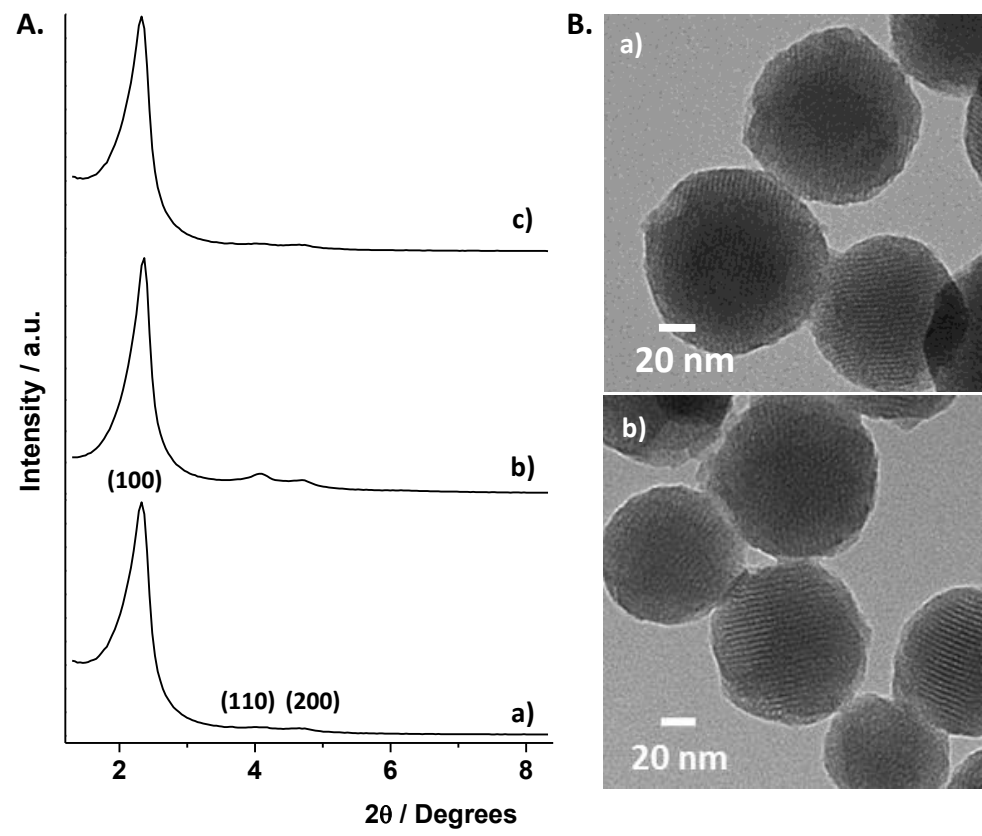

Figure 1. A: Powder X-ray patterns of the solids: (a) MCM-41 as synthesized, (b) calcined MCM-41 and (c) solid S1. B: Representative TEM images of: (a) calcined MCM-41 sample and (b) solid S1.

Table 1. Main structural properties calculated from TEM, PXRD and $\mathrm{N}_{2}$ adsorption-desorption analysis of MCM-41 and S1.

\begin{tabular}{cccc}
\hline Sample & $\begin{array}{c}\mathrm{S}_{\mathrm{BET}} \\
\left(\mathrm{m}^{2} \mathrm{~g}^{-1}\right)\end{array}$ & $\begin{array}{c}\text { Pore volume } \\
\left(\mathrm{cm}^{3} \mathrm{~g}^{-1}\right)\end{array}$ & $\begin{array}{c}\text { Pore diameter } \\
(\mathrm{nm})\end{array}$ \\
\hline MCM-41 & 888 & 0.72 & 2.59 \\
S1 & 227 & 0.14 & - \\
\hline
\end{tabular}


To assess the feasibility of the proposed sensing paradigm, controlled release experiments from S1-Ars-3 in the presence of As(III) dissolved in aqueous media were carried out. In a typical experiment, $150 \mu \mathrm{g}$ of S1-Ars-3 were suspended in 1.5 mL of HEPES buffer (pH 7.2) and the suspension was divided in two fractions. The first fraction was diluted to $900 \mu \mathrm{L}$ with Milli-Q water as a control experiment. On the other hand, the second fraction was diluted with a stock solution of As(III) in Milli-Q water to reach a concentration of $100 \mathrm{ppb}$ of As(III) in a final volume of $900 \mu \mathrm{L}$. Both suspensions were stirred at room temperature and aliquots were taken at certain programmed times $(0,5,10,1525,30$ and $35 \mathrm{~min})$. The aliquots were centrifuged to eliminate the solid, and cargo release was evaluated by measuring the fluorescence emission of rhodamine $\mathrm{B}$ in the supernatant centred at $572 \mathrm{~nm}\left(\lambda_{\mathrm{ex}}=555\right)$. Delivery kinetic profiles of rhodamine B both in the presence and absence of As(III) are shown in Figure 2a. As could be seen, in the absence of the target analyte, solid S1-Ars-3 showed a very small rhodamine B release of $1.2 \mu \mathrm{mol} / \mathrm{g}$ S1-Ars-3 (ca. 5\% of the maximum dye delivered after $25 \mathrm{~min}$ ) indicating a tight pore closure. However, in the presence of $100 \mathrm{ppb}$ of As(III), a clear rhodamine B delivery was observed that reaches nearly $80 \%$ (ca. $22 \mu \mathrm{mol} / \mathrm{g}$ S1-Ars-3) of the maximum dye delivered after $c a .25$ min. The observed rhodamine B release was a result of the As(III)-induced displacement of the capping aptamer from the nanoparticles' surface. As(III) has been reported to form highly stable complexes with Ars-3 through strong hydrogen bond interactions of the hydroxyl groups of aqueous As(III) with amine groups of nucleobases in the aptamer chain. ${ }^{27}$ 


\section{a)}

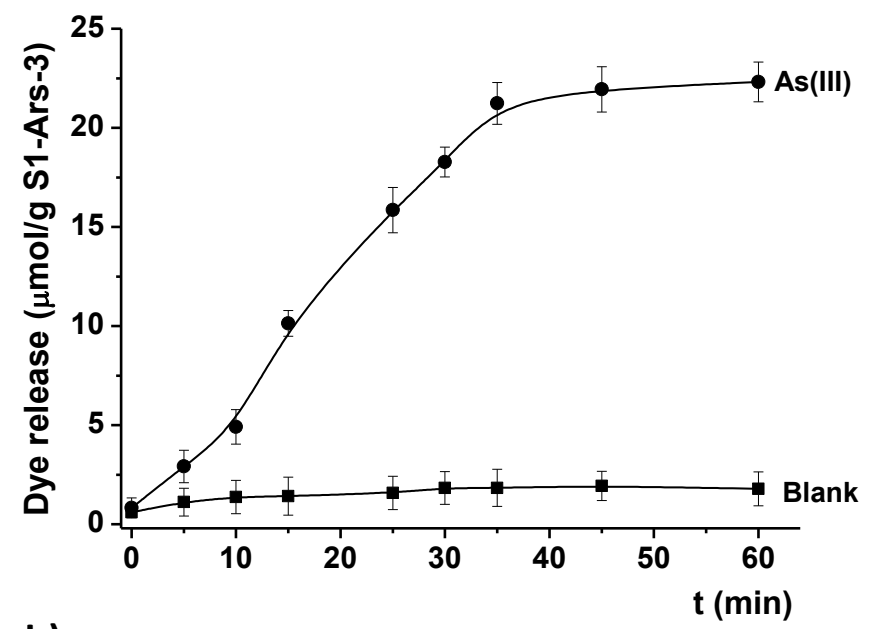

b)

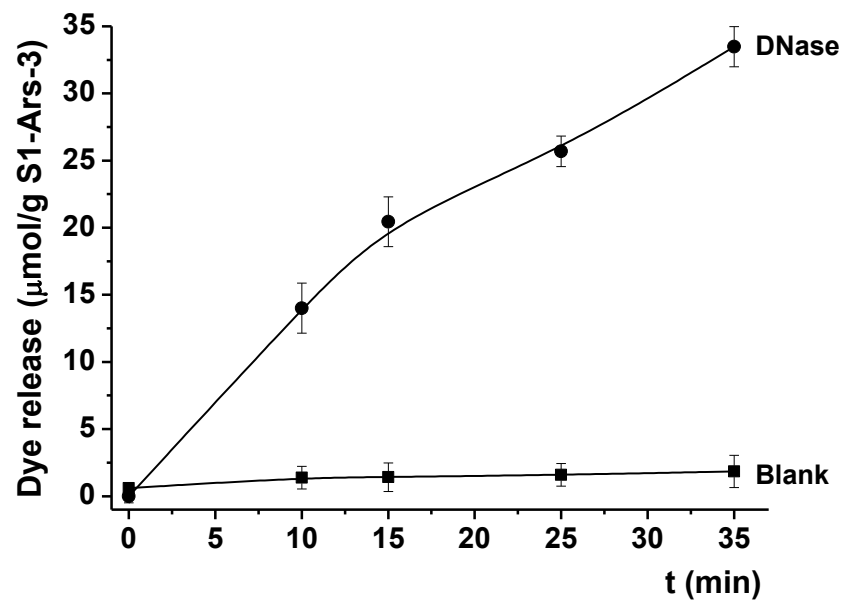

Figure 2. (a) Release profile of rhodamine B from S1-Ars-3 in the absence and in the presence of As(III) (100 ppb) in HEPES buffer (pH 7.2). (b) Delivery of rhodamine B from solid S1-Ars3 in the absence and in the presence of $55 \mathrm{U} / \mu \mathrm{L}$ of DNAse I enzyme.

To reinforce the demonstration of the proper interaction of aptamer Ars-3 with solid S1, release experiments in presence of DNAse I enzyme were carried out. Results in the presence of DNAse I enzyme, showed a remarkable dye release from solid S1-Ars-3, indicating the essential role of the Ars-3 aptamer in the capping mechanism (see Figure 2b). 
In a further step, the sensitivity of the prepared nanocarrier towards As(III) was evaluated. In this experiment dye released after $35 \mathrm{~min}$ from S1-Ars-3 in the presence of different amounts of As(III) was studied. As seen in Figure 3 rhodamine B delivery was proportional to the As(III) concentration, which is in agreement with the proposed uncapping protocol involving the displacement of Ars-3 from the surface of solid S1-Ars-3. From these experiments a linear response in the 4-60 ppb As(III) concentration range was found. Furthermore, a limit of detection (LOD) as low as $0.9 \mathrm{ppb}$ was determined. This LOD is $c a .10$-fold below the EPA toxicity level of arsenic in drinking water $(10 \mathrm{ppb}){ }^{2}$ The calculated LOD indicates a noteworthy sensitivity of the nanoprobe, and confers our system a competitive basis with other As(III) aptameric sensors based on colorimetric, ${ }^{28-30}$ fluorometric $^{31}$ and resonance Rayleigh scattering ${ }^{28,33}$ measurements (see Table S5 in the Supporting Information).

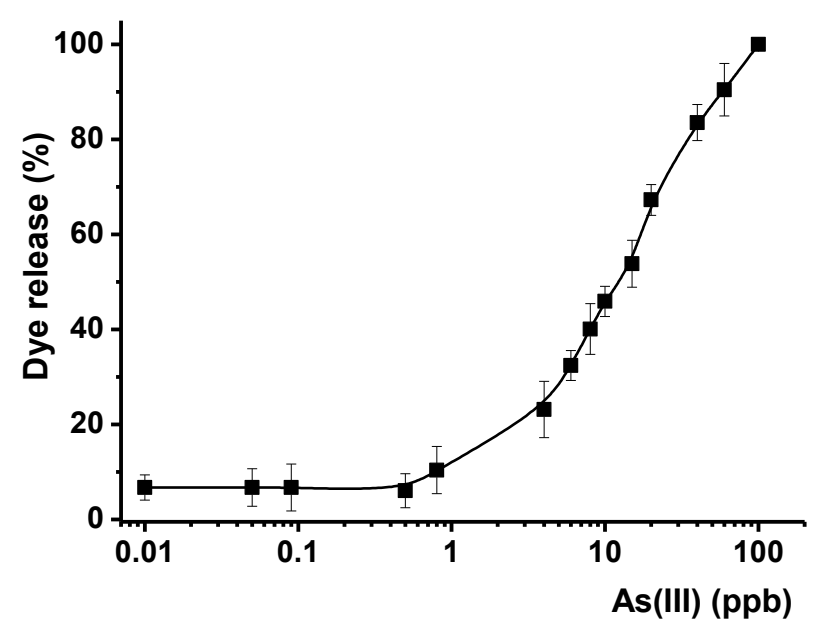

Figure 3. Release of rhodamine B from solid S1-Ars-3 in the presence of increasing quantities of As(III) in HEPES buffer (pH 7.2) after 35 min of addition.

The next step was to verify the selectivity of S1-Ars-3 solid. For this purpose, cargo release from S1-Ars-3 was tested in water containing 50 ppb of $\operatorname{As}(\mathrm{III}), \operatorname{As}(\mathrm{V}), \operatorname{Ag}(\mathrm{I}), \mathrm{Pb}(\mathrm{II}), \mathrm{K}(\mathrm{I})$, 
$\mathrm{Na}(\mathrm{I}), \mathrm{Fe}(\mathrm{III}), \mathrm{Fe}(\mathrm{II}), \mathrm{Mg}(\mathrm{II})$ and $\mathrm{Ca}(\mathrm{II})$ cations. As shown in Fig. 4, As(III) is the only cation able to induce a remarkable payload delivery, whereas other metal ions tested induced poor pore opening and negligible dye release. This observation corroborates the selective As(III)-aptamer interaction as the main mechanism of the fluorogenic response observed.

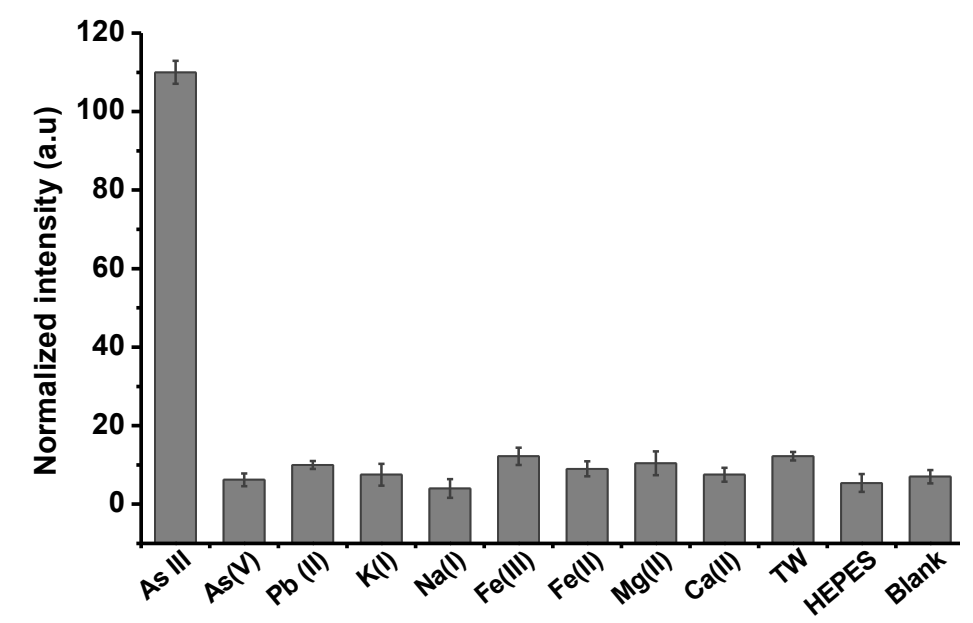

Figure 4. Release or rhodamine B from S1-Ars-3 in the presence of different ions at a concentration of $50 \mathrm{ppb}$ after $35 \mathrm{~min}$ of addition. Two control experiments were carried out with Milli-Q water (blank), with HEPES buffer at pH 7.2 (HEPES), and with tap water (TW).

Motivated by the excellent properties displayed by S1-Ars-3, we took a step forward and tested the performance of the capped material for the determination of As(III) in an environment conditions. With this aim, in a typical experiment, tap water samples were spiked with 4, 6 and $10 \mathrm{ppb}$ of As(III), respectively and the concentration of As(III) was determined using a calibration curve obtained using S1-Ars-3 solid. Table 2 shows the concentration of As(III) determined in the spiked samples using S1-Ars-3. Recovery levels were within the 91109\%range indicating the suitability of S1-Ars-3 nanoparticles for the determination of As(III) ions in a real environment. 
Table 2. Determination of As(III) in tap water samples.

\begin{tabular}{ccc}
\hline As(III) added (ppb) & As(III) found (ppb) & Recovery (\%) \\
\hline 4 & $3.6 \pm 0.1$ & 91 \\
6 & $6.5 \pm 0.2$ & 108 \\
10 & $11.0 \pm 1.2$ & 109
\end{tabular}

In summary, we have successfully developed a novel fluorogenic sensing probe for As(III) by combining MSNs with aptamers as capping agents. The sensing mechanism relies on the specific interaction between the capping aptamer (Ars-3) and the As(III). The prepared nanoprobe showed a remarkable LOD of $0.9 \mathrm{ppb}$. In addition, the hybrid nanomaterial allowed accurate As(III) determination in tap water samples. As far as we know, this is the first time that aptamercapped mesoporous supports are used for the detection of As(III). The presented approach is simple and undemanding, and we believe that possesses the potential of finding application in environmental analysis protocols.

\section{ASSOCIATED CONTENT}

Supporting Information. The following files are available free of charge: Synthesis and characterization of the sensory materials and controlled release experiences with As(III) (PDF). AUTHOR INFORMATION

\section{Corresponding Author}

*Ramón Martínez-Máñez: rmaez@qim.upv.es

*Dmitry G. Shchukin: D.Shchukin@liverpool.ac.uk

\section{ACKNOWLEDGMENTS}


The authors thank the financial support from the Spanish Government (projects MAT201564139-C4-1-R and AGL2015-70235-C2-2-R) and the Generalitat Valenciana (project GVA/2014/13). M.O. is grateful to the Universitat Politècnica for her PhD grant. A.B. thanks the Spanish Government for the finantial support "Juan de la Cierva-Incorporación" (IJCI-201421534). C.C. thanks the Generalitat Valenciana for her postdoctoral contract VALi+D.

\section{REFERENCES}

(1) Mandal, B. K.; Suzuki, K. T. Arsenic Round the World: A Review. Talanta 2002, 58, 201-235.

(2) United States Environ. Prot. Agency. Arsen. Clarifications to Compliance New Source Monit. Rule A Quick Ref. Guide. EPA 816-F-01-004; US EPA Washington, DC, 2001; http//www.epa.gov/safewater/arsenic/compliance.html (accessed 10/ 2015).

(3) Yang, B.; Chen, X.; Liu, R.; Liu, B.; Jiang, C. Target Induced Aggregation of Modified Au@Ag Nanoparticles for Surface Enhanced Raman Scattering and its Ultrasensitive Detection of Arsenic in Aqueous Solution. RSC Adv. 2015, 5, 77755-77759.

(4) Bissen, M.; Frimmel, F. H.; Ag, C. Arsenic - A Review . Part I: Occurrence, Toxicity, Speciation, Mobility. Acta Hydrochim. Hydrobiol. 2003, 31, 9-18.

(5) Tuzen, M.; Saygi, K. O.; Karaman, I.; Soylak, M. Selective Speciation and Determination of Inorganic Arsenic in Water, Food and Biological Samples. Food Chem. Toxicol. 2010, 48, 4146.

(6) Aggett, J.; Aspell, A. C. The Determination of Arsenic(III) and Total Arsenic by AtomicAbsorption Spectroscopy. Analyst 1976, 101, 341-347. 
(7) Yan, X.-P.; Kerrich, R.; Hendry, M. J. Determination of (Ultra)trace Amounts of Arsenic(III) and Arsenic(V) in Water by Inductively Coupled Plasma Mass Spectrometry Coupled with Flow Injection On-Line Sorption Preconcentration and Separation in a Knotted Reactor. Anal. Chem. 1998, 70, 4736-4742.

(8) Zhou, Y.; Huang, X.; Liu, C.; Zhang, R.; Gu, X.; Guan, G.; Jiang, C.; Zhang, L.; Du, S.; Liu, B.; Han, M. -Y.; Zhang, Z. Color-Multiplexing-Based Fluorescent Test Paper: DosageSensitive Visualization of Arsenic(III) with Discernable Scale as Low as 5 ppb. Anal. Chem. 2016, $88,6105-6109$.

(9) Baglan, M.; Atilgan, S. Selective and Sensitive Turn-on Fluorescent Sensing of Arsenite Based on Cysteine Fused Tetraphenylethene with AIE Characteristics in Aqueous Media. Chem. Commun. 2013, 49, 5325-5327.

(10) Ezeh, V. C.; Harrop, T. C. Synthesis and Properties of Arsenic(III)-Reactive CoumarinAppended Benzothiazolines: A New Approach for Inorganic Arsenic Detection. Inorg. Chem. 2013, 52, 2323-2334.

(11) Li, M.; Gou, H.; Al-Ogaidi, I.; Wu, N. Nanostructured Sensors for Detection of Heavy Metals: A Review. ACS Sustainable Chem. Eng. 2013, 1, 713-723.

(12) Zheng, Z.; Huang, X.; Shchukin, D. A Cost-Effective pH Sensitive Release System for Water Source pH Detection. Chem. Commun. 2014, 50, 13936.

(13) Sancenón, F.; Pascual, L.; Oroval, M.; Aznar, E.; Martínez-Máñez, R. Gated Silica Mesoporous Materials in Sensing Applications. ChemistryOpen 2015, 4, 418-437. 
(14) Aznar, E.; Oroval, M.; Pascual, L.; Murguía, J. R.; Martínez-Máñez, R.; Sancenón, F. Gated Materials for On-Command Release of Guest Molecules. Chem. Rev. 2016, 116, 561-718.

(15) Zheng, Z.; Huang, X.; Schenderlein, M.; Moehwald, H.; Xu, G.; Shchukin, D. G. Bioinspired Nanovalves with Selective Permeability Andand pH Sensitivity. Nanoscale 2015, 7, 2409-2416.

(16) Zheng, Z.; Huang, X.; Schenderlein, M.; Borisova, D.; Cao, R.; Möhwald, H.; Shchukin, D. Self-Healing and Antifouling Multifunctional Coatings Based on $\mathrm{pH}$ and Sulfide Ion Sensitive Nanocontainers. Adv. Funct. Mater. 2013, 23, 3307-3314.

(17) Choi, Y. L.; Jaworski, J.; Seo, M. L.; Lee, S. J.; Jung, J. H. Controlled Release Using Mesoporous Silica Nanoparticles Functionalized with 18-Crown-6 Derivative. J. Mater. Chem. 2011, 21, 7882-7885.

(18) Li, F.; Zhang, H.; Wang, Z.; Newbigging, A. M.; Reid, M. S.; X.-F. L.; X. C. Le. Aptamers Facilitating Amplified Detection of Biomolecules. Anal. Chem. 2015, 87, 274-292.

(19) Oroval, M.; Coronado-Puchau, M.; Langer, J.; Sanz-Ortiz, M. N.; Ribes, Angela; Aznar, E.; Coll, C.; Marcos, M. D.; Sancenón, F.; Liz-Marzán, L. M.; Martínez-Máñez, R. Surface Enhanced Raman Scattering and Gated Materials for Sensing Applications: The Ultrasensitive Detection of Mycoplasma and Cocaine. Chem. Eur. J. 2016, 13488-13495.

(20) Özalp, V. C.; Çam, D.; Hernandez, F. J.; Hernandez, L. I.; Schäfer, T.; Öktem, H. a. Small Molecule Detection by Lateral Flow Strips via Aptamer-Gated Silica Nanoprobes. Analyst 2016, 141, 2595-2599. 
(21) He, X.; Zhao, Y.; He, D.; Wang, K.; Xu, F.; Tang, J. ATP-Responsive Controlled Release System Using Aptamer-Functionalized Mesoporous Silica Nanoparticles. Langmuir 2012, 28, $12909-12915$.

(22) Wen, Y.; Xu, L.; Li, C.; Du, H.; Chen, L.; Su, B.; Zhang, Z.; Zhang, X.; Song, Y. DNABased Intelligent Logic Controlled Release Systems. Chem. Commun. 2012, 48, 8410-8412.

(23) Chen, L.; Wen, Y.; Su, B.; Di, J.; Song, Y.; Jiang, L. Programmable DNA Switch for Bioresponsive Controlled Release. J. Mater. Chem. 2011, 21, 13811-13811.

(24) Oroval, M.; Climent, E.; Coll, C.; Eritja, R.; Aviñó, A.; Marcos, M. D.; Sancenón, F.; Martínez-Máñez, R.; Amorós, P. An Aptamer-Gated Silica Mesoporous Material for Thrombin Detection. Chem. Commun. 2013, 49, 5480-5482.

(25) Argyo, C.; Weiss, V.; Bräuchle, C.; Bein, T. Multifunctional Mesoporous Silica Nanoparticles as a Universal Platform for Drug Delivery. Chem. Mater. 2014, 26, 435-451.

(26) Coll, C.; Mondragón, L.; Martínez-Máñez, R.; Sancenón, F.; Marcos, M. D.; Soto, J.; Amorós, P.; Pérez-Payá, E. Enzyme-Mediated Controlled Release Systems by Anchoring Peptide Sequences on Mesoporous Silica Supports. Angew. Chem. Int. Ed. 2011, 50, 2138-2140.

(27) Kim, M.; Um, H. J.; Bang, S.; Lee, S. H.; Oh, S. J.; Han, J. H.; Kim, K. W.; Min, J.; Kim, Y. H. Arsenic Removal from Vietnamese Groundwater Using the Arsenic-Binding DNA Aptamer. Environ. Sci. Technol. 2009, 43, 9335-9340.

(28) Wu, Y.; Liu, L.; Zhan, S.; Wang, F.; Zhou, P. Ultrasensitive Aptamer Biosensor for Arsenic(III) Detection in Aqueous Solution Based on Surfactant-Induced Aggregation of Gold Nanoparticles. Analyst 2012, 137, 4171-4178. 
(29) Zhan, S.; Yu, M.; Lv, J.; Wang, L.; Zhou, P. Colorimetric Detection of Trace Arsenic(III) in Aqueous Solution Using Arsenic Aptamer and Gold Nanoparticles. Aust. J. Chem. 2014, 67, $813-818$.

(30) Divsar, F.; Habibzadeh, K.; Shariati, S.; Shahriarinour, M. Aptamer Conjugated Silver Nanoparticles for the Colorimetric Detection of Arsenic Ions Using Response Surface Methodology. Anal. Methods 2015, 7, 4568-4576.

(31) Ensafi, A. A.; Kazemifard, N.; Rezaei, B. A Simple and Sensitive Fluorimetric Aptasensor for the Ultrasensitive Detection of Arsenic(III) Based on Cysteamine Stabilized CdTe/ZnS Quantum Dots Aggregation. Biosens. Bioelectron. 2015, 77, 499-504.

(32) Kaur, H.; Kumar, R.; Babu, J. N.; Mittal, S. Advances in Arsenic Biosensor Development - A Comprehensive Review. Biosens. Bioelectron. 2015, 63, 533-545.

(33) Wu, Y.; Zhan, S.; Xing, H.; He, L.; Xu, L.; Zhou, P. Nanoparticles Assembled by Aptamers and Crystal Violet for Arsenic(III) Detection in Aqueous Solution Based on a Resonance Rayleigh Scattering Spectral Assay. Nanoscale 2012, 4, 6841-6849. 


\section{Graphic for manuscript}
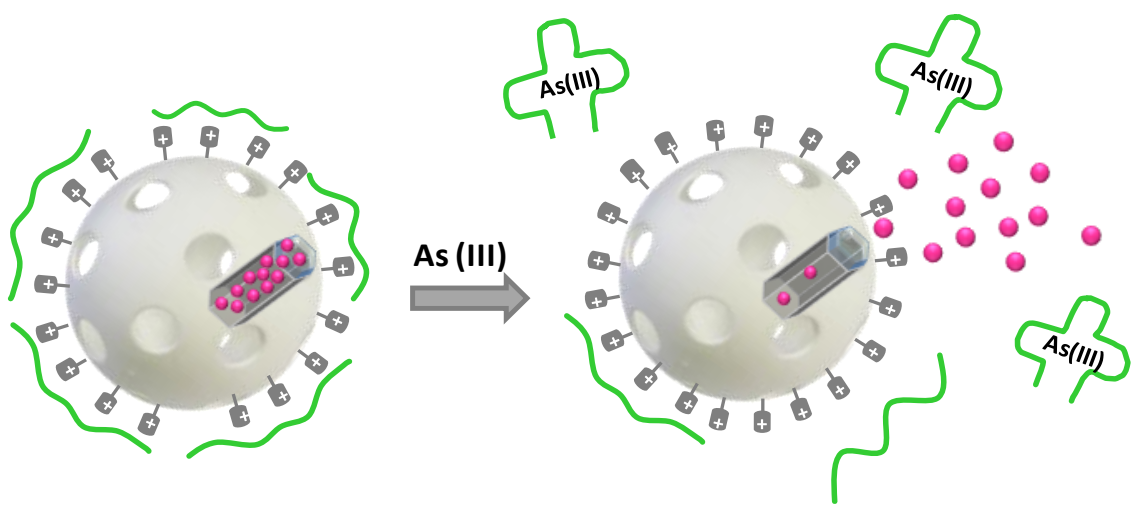